\title{
Merging the Biological and Cognitive Processes of Sleep and Screens
}

\author{
Heather Cleland Woods ${ }^{1} \cdot$ Holly Scott ${ }^{1}$
}

Published online: 31 July 2019

(C) The Author(s) 2019

\begin{abstract}
Purpose of Review Screens are a permanent feature of life today and we have reached an interesting juncture with different research agendas investigating the biological and cognitive aspects of screen use separately. This review argues that it is timely and indeed essential that we bring together these research areas to fully understand both positive and negative aspects of screen use.

Recent Findings More recent work is starting to take a more cohesive approach to understanding how device use pre-bedtime can impact our sleep by including both light and content in their experimental protocols which is a welcome development leading to a more nuanced understanding of both biological and cognitive processes.

Summary We call for an open and collaborative approach to gain momentum in this direction of acknowledging both biological and cognitive factors enabling us to understand the relative impacts of both whilst using screens with regard to both light and content.
\end{abstract}

Keywords Sleep $\cdot$ Blue light $\cdot$ Circadian $\mathrm{rhythm} \cdot$ Screens $\cdot$ Social $\cdot$ Melatonin

\section{Introduction}

Screens are a permanent feature of lives today as a gateway to browsing and sharing information and social interaction. Research to date has attempted to understand the impact of screens on our sleep and wellbeing by either looking at the impact of blue light isolated from content or content isolated from the physiological impact of blue light. The aim of this review is to pull together these two areas of sleep research which have been investigated separately, with a recent exception, where we will argue that it is a misrepresentation to consider them as separate entities. Blue light has been highlighted as having a significant negative effect on sleep within the past decade in the discussion around screen time; however, we aim to show that more recent work into the social aspects of online engagement has led us to understand the interactive nature of social media use and therefore bringing us to an important reflection point in sleep and technology

This article is part of the Topical Collection on Sleep and Technology

Heather Cleland Woods

Heather.Woods@glasgow.ac.uk

1 School of Psychology, University of Glasgow, 62 Hillhead Street, Glasgow G12 8QB, UK research where we can recognise the complexities and sophistication of this online social interaction. This, along with research into the impact of blue light exposure on the biological processes involved in sleep, will enable us to include light as one of a range of relevant factors on how we consume media with the broader range of factors inclusive of social interaction and cognitive arousal from content. It is essential that we have a full appreciation of the holistic nature of interactions with screens, inclusive of all biological, social and cognitive aspects, to enable us to make informed decisions in policy and practice on screens and sleep. Sleep is known to change with age and adolescence is a developmental stage where circadian phase is vulnerable to change [1] as well as having high screen and social media use [2]. This provides an interesting profile to understand the interactions between these factors so this review will predominantly focus on research from the sleep, circadian and social media fields working with adolescents.

\section{The Perfect Storm Online}

The generic term "screen time" has been widely discussed in the adolescent sleep field, where it has also been established for a significant time that adolescents experience a circadian delay [3]. In relation to screen use, Carskadon [4] proposed that development of bioregulatory mechanisms alongside 
psychosocial factors (such as seeking increased independence and bedtime autonomy, which can provide opportunity for pre-sleep activities such as social media use) resulted in a 'perfect storm' of short, ill-timed and inadequate sleep in many teens. There is tension between two biological systems during adolescence. The sleep/wake homeostatic process, with which the "pressure" for sleep builds across the day, and the circadian system which dictates the timing of many physiological and behavioural rhythms, including alertness. Findings indicated that for more mature adolescents, the recovery process did not accelerate, whereas the sleep pressure accumulation process decelerated, from which Carskadon [4] inferred an ease of staying awake longer. Findings reviewed by Carskadon [4] and colleagues indicated a delay in this internal clock and a slowing down of the sleep pressure accumulation as adolescents mature resulting in alertness and bedtimes later into the evening and night and rising later into the morning when possible. This has led to the phrase social jet lag with sleep being out of sync with this internal rhythm and that sleep debt being paid at weekends [5]. Crowley et al. [3] offer a conclusion that evening light from devices with screens may activate this sleep phase-delaying component of the internal clock which highlights the need for a pause for thought in relation to this field to enable a robust research basis for a topic of concern and prolific discussion for our adolescents, parents, teachers as well as policymakers. We have a situation where parents, teachers and public bodies are voicing concern about adolescents and children getting enough sleep [6••] and the perception that this resulting deficit is impacting their ability to be alert and effective in school. By trying to regulate and maintain control over adolescents who are not ready for sleep provides an ideal opportunity for screen-based media use opening up the potential for further delay to sleep onset resulting from exposure to stimulating blue light and content. Bookend this delayed sleep onset due to changes in homeostatic pressure and circadian phase with school start times earlier than suited to adolescent sleep then we have a pressurized situation with sleep paying the price.

\section{Media as a Source of Screen Light}

The biological and physiological processes behind light and the biological clock have been outlined in detail elsewhere [7] so we shall focus on how light, screens and social media link together before continuing onto outline the research profile. To translate circadian from Latin is about a day ( circa $=$ about, dia $=$ day) which represents the 24-h day cycle that most humans and other animals adhere to even when free running without external input. This circadian rhythm is governed by a group of cells in our anterior hypothalamus above the optic chiasm called the suprachiasmatic nucleus $(\mathrm{SCN})$ which is our internal clock or internal pacemaker which entrains us to this 24-h cycle. The most influential synchronizer or Zeitgeber which provides external input for this circadian process is light. When light is absent or extended, as documented in experimental protocols, the circadian rhythm of melatonin, a hormone produced by the pineal gland which follows a circadian rhythm with low levels during the day and high levels at night, free-runs. This means that, as it is no longer synchronized with the environmental light-dark cycle, it becomes out of phase with this environmental cycle $[8,9]$. Light is important in melatonin production as cells within the retina called intrinsically photosensitive retinal ganglion cells (ipRGCs) contain melanopsin that is expressed in a small subset of cells representing 1-2\% of all retinal ganglion cells. Melanopsin is sensitive to blue light (i.e. wavelengths ranging from 460 to $480 \mathrm{~nm}$ ) and that is fundamental for the functioning of the circadian system and for SCN entrainment. The light signal received by the retina is transmitted to the SCN ending up at the pineal gland which secretes melatonin [9].

In terms of media use, this blue light-sensitive melanopsin photoreceptor has received a lot of attention as handheld devices such as smartphones which emit light will include blue light as part of the light spectrum. White light includes light from across the spectrum (including blue between 460 and $480 \mathrm{~nm}$ ) but as we know the arousing effects of blue light exposure, it is essential to examine the effects of sources of these wavelengths on this bioregulatory system which is responsible for our sleep onset and may be disrupted by our use of devices. Light-emitting diode (LED) screens, which handheld devices such as smartphones and tablets have, are reported to emit a high level of this blue light and evidence has been provided suggesting that this has a negative impact on sleep which may be particularly relevant for adolescents who are vulnerable to circadian developmental delay.

\section{Current Understanding}

The research on the impact of light on sleep is prevalent and great care has been paid to the quality and precision in which participants are exposed to the light and wavelengths of interest. Cajochen et al. [10] have shown that a 5-h exposure to a (white) light-emitting diode (LED) backlit computer screen significantly suppressed melatonin and enhanced performance compared with a non-LED backlit screen. Their results showed that although melatonin levels were still rising over the course of the night, they did not rise as steeply and were delayed compared with those exposed to a non-LED screen. It is highly relevant that simply the light from the LED source in our screens can delay this biological process, but if we evaluate this study which asks a relevant question about the impact of the strongest Zeitgeber, with regard to sleep there are a few considerations. Firstly, the melatonin is delayed and not attenuated - it starts to rise later and is decreased at 4 time points. It would be of interest to get a clearer handle on the effect on melatonin in terms of effect size to enable 
contextualisation combined with cognitive factors and reallife impact on sleep and wellbeing. With shift work being highly prevalent in today's society which is drawing attention of governing bodies [11, 12], this work does have positive implications in terms of keeping work force safe and alert with the other side of the argument focusing on a decrease in alertness facilitating sleep onset.

Timing and duration of screen exposure is an interesting aspect to consider. Studies such as Cajochen et al. [10] and Chang et al. [13] use a consolidated time for screen exposure pre-bedtime. The duration of exposure has been argued to be modest with Cajochen et al. [10] using a 5-h screen exposure and Chang et al. [13] using 4-h exposure compared with a data from the USA which shows US 8- to 18-year-olds devote an average of $7 \mathrm{~h}$ and $38 \mathrm{~min}$ to using entertainment media across a typical day (more than $53 \mathrm{~h} /$ week) [14]. This leads us to the time on screen aspect of the screen time debate. How representative is a consolidated $5 \mathrm{~h}$ of screen viewing? Cajochen et al. [10] acknowledge that many adolescents use multiple screens for different activities at any one time. For example, one may be writing on a laptop whilst communicating with friends on communication apps on different devices.

Effect size and ecological validity of these paradigms are also raised in Chang et al. [13] where participants with a mean age of 25 years passively read a self-selected text from an iPad for approx. 4 consolidated hours pre-bedtime which led to a 10-min mean difference in sleep onset and no difference in total sleep time, sleep efficiency or duration of non-REM sleep. Again, melatonin level was delayed but still followed same pattern as seen in Cajochen et al. [10]. It is interesting to consider the extent to which a 10-min (yet statistically significant) sleep onset delay from consolidated pre-bedtime light exposure really impacts our teens' wellbeing and health in the long term compared with other relevant determinants of sleep time, e.g. school start times or pre-sleep cognitive arousal due to fear of peer exclusion. This research area not only caught the attention of the media and public which outlines the reach of these findings but also highlighted the importance of providing context around our findings.

Orben and Przybylski [15] demonstrate the positive influence sleep has on adolescent wellbeing is significantly larger that technology use alongside eating breakfast, fruit and vegetables. Within this context, it is frustrating to see such pathologizing language as that used around technology use in the literature where use of terms such as addiction and disorder immediately pathologizes all night-time online activity without acknowledging the diversity of cognitive factors behind engagement [16]. To link back to the previous effect size discussion, it would be an informative future research agenda to evaluate the impact of 10-min sleep onset delay compared with adolescents feeling excluded or anxious due to disengagement of continuing online interactions. Despite the delay of melatonin production, we still see increases, albeit delayed, in both the Cajochen et al. [10] and Chang et al. [11] studies, so understanding the effect size of this delay is important as well as the context within which these findings are discussed whilst addressing pre-sleep arousal related to content and context leading to difficulty disengaging from social interaction.

\section{Screen Light in the Context of Real World Social Media Use}

Our understanding of the pressures building in adolescent's 'perfect storm' alongside evaluation of the impact of blue light on sleep considers important aspects of media as a source of disruption to sleep but requires us to reflect on social and cognitive aspects as we know that teenage social media use has increased almost tenfold increase in recent years [17]. Currently, 95\% 13-17-year-olds have access to smartphones and $45 \%$ admit to being online almost constantly [2]. Recent UK data has shown that $20 \%$ of the adolescents involved in the Millennium Cohort Study used social media for $5 \mathrm{~h}$ or more and that this resulted in them being more likely than comparable typical users (1-3 h per day, $31.6 \%$ ) to report late sleep onset and wake times on school days and frequent difficulties with night-time awakenings [18]. To date, screen time has been used as a generic measure of use without explicitly stating which activity is being undertaken, for example in a group chat with friends, accessing homework assessments, actively or passively browsing content. Social media is the most prevalent activity when teens use screens and this has been linked to current increases in teenage mental health problems, such as depression [19] and lowered self-esteem [20]. Consequently, there has been a drive to understand the reason for such high usage rates and the implications for young people. In doing so, the term screen time is becoming less informative as there is increasing recognition of the rich and diverse nature of online experiences. Furthermore, there is still little consensus on what defines platforms as 'social media' [21], since descriptions have evolved from including only professional networking sites, such as LinkedIn (2005), to encompassing more personal sites, like Facebook (17). Adolescents appear to engage with YouTube, Instagram and Snapchat most frequently (2) with the latter two being media of a social nature enabling users to share images and interaction with others.

Bartel and Gradisar [22••] raise the highly relevant questions of 'how' and 'how much' technology use impacts sleep acknowledging the contributions of previous work on biological and bedtime practices. They move this discussion on by acknowledging that there are a number of other factors relevant to this link between screens and sleep and propose a revised model including moderating factors and potential mechanisms. These include developmental considerations such as risk taking as it is known not only that adolescents can evaluate and respond to risk in different ways from 
younger children and adults especially when external factors such as the presence of peers has influence [23] but also that teens are more impulsive to positive social cues [24]. It is therefore important that we acknowledge this social nature of technology use alongside the exposure to light.

\section{A Holistic Approach Moving Forward}

More recent work has attempted to bring a more holistic approach to the field by investigating both light and content with the argument that light and stimulating content may be working on biological and cognitive mechanisms simultaneously. Bowler and Bourke [25] ran a 4-night study with 30 participants browsing own or mock Facebook pages on iPads over two conditions with a blue filter or an amber filter. The mock Facebook page consisted of liked pages of companies that were not targeted to the participant's age range, e.g. FisherPrice toys. Significant interactions on arousal and light with blue filter and low arousal on the mock Facebook page showed better sleep through longer sleep duration, shorter sleep onset latency and better daytime dysfunction. This study highlights the impact that both light and content simultaneously can have on sleep and related outcomes following a short duration of exposure as participants were instructed to only 15- to 30-min exposure in the hour before bed. Showing blue light filtering only had an effect when the content being viewed was not personally relevant and low arousing further strengthens the argument, through a new experimental protocol, that both biological and cognitive aspects need to be considered.

This more recent light exposure paradigm was significantly shorter than that in the previous studies. Bowler and Burke [25] exposed the participants to the devices and therefore light and content for 15 to $30 \mathrm{~min}$ and cite Horne, Donlon and Arendt [26] as having seen such fast effects previously. This raises the question about the length of exposure - are the effects we see in Cajochen and Chang due to prolonged exposure to blue light as they use pre-sleep paradigms lasting $4 \mathrm{~h}$ [13] and $5 \mathrm{~h} \mathrm{[10].} \mathrm{Also,} \mathrm{the} \mathrm{activities} \mathrm{seen} \mathrm{in} \mathrm{all} \mathrm{these} \mathrm{studies}$ are very passive with participants being exposed to light or passively being presented with information rather than being active and posting, liking and communicating along with browsing content. McNee and Cleland Woods [27] have recently started to pursue this line of thought around activities online rather than just time on screen which highlights an association between social comparison orientation, individuals driven to evaluate their progress and standing on various aspects of their lives against similar peers [28], night-time social media use, mental wellbeing, and sleep in female users of Facebook and Instagram $(N=60)$. Social comparison orientation, rumination tendency, sleep health and night-time social media use were examined using online questionnaire measures. Social comparison orientation was found to be positively associated with night-time social media use and rumination tendency. In addition, rumination was highlighted as a significant mediator of the relationship between social comparison behaviour and poor sleep - suggesting that the behaviours we engage in online may influence sleep through a cognitive pathway.

Pre-sleep arousal is a cognitive aspect of sleep and insomnia which has been recognised for a number of years [29] and has relevance to understanding the cognitive aspects of social media's impact on sleep. Scott and Cleland Woods [30] highlight two parallel pathways that predict shorter sleep duration stemming from a shared underlying cognitive driver; fear of missing out (FOMO). We saw separate behavioural and cognitive components, with FOMO driving adolescents to actively engage more in social media behaviours at night (the behavioural component) and also feel more alert in bed (the cognitive component). This work highlights the social nature of media use at night and can account for delayed disengagement from devices which is important to acknowledge along with the effects of blue light from screens as biological interference with ideal sleep onset. This work also highlights the importance of taking a step back from placing the screen at the centre of the issue and addressing the content displayed on the screen and the resulting interactions which result in behaviours such as night-time social media use leading to delayed bedtimes and restricted sleep opportunity for those schoolaged adolescents needing to get to school on time $[22 \bullet \bullet, 31$, 32]. Sleep onset and therefore duration can also be impacted by the cognitive arousal from reluctance and potentially fear of missing out on group communication and sharing of information which is not necessarily related to time spent on social media in bed [30]. This reluctance to be the group member missing out is not a new phenomenon as peer acceptance is an important part of adolescent development, but social media takes this out of school and into the bedroom and the possibility of delayed disengagement at bedtime. Bartel and Gradisar [22••] also raise the point that work to date had perhaps focused on the unidirectional relationship between screens and sleep on the premise of screens cause poor sleep and it is time we acknowledge that our adolescents may be using screens due to sleep issues or timing differences with their families already established. This again highlights that we are at a point in time where we can bring together what is seen in clinic with our research questions and practices to pull a comprehensive research agenda together to support our teens.

We need to bring biological and social factors which impact sleep together by understanding the limitations within this complex field. The combination of biological and psychosocial factors continues from Carskadon's model as it is not only the light from these devices that needs to be considered but also the content and context of viewing material on the devices. Chang et al. [13] had participants looking at relaxing 
reading material but this picture may be unrealistic as our research suggests that communication with peers is a significant factor in delaying disengagement from devices at night. Our model addresses both behavioural and cognitive factors of night-time social media use [30] as it demonstrates that FOMO is the cognitive driver for night-time social media use and impacts sleep onset latency (subjectively reported) negatively via pre-sleep cognitive arousal.

This model suggests that looking at light from devices is only part of the story as cognitive factors are essential to be considered within the recognition of device use as often a social interaction rather than passive browsing. When we relate this to Carskadon's model [3, 4] with psychosocial factors such as social networking and bedtime autonomy recognised as pressures on sleep in adolescents, we can build a sophisticated model of night-time pressures on adolescent sleep that builds on the impact of blue light on the biological processes and includes the social opportunities felt by our teens which may now present within the bedroom. Content and context influencing cognitions and behaviours are just as relevant as light influencing the biology of circadian phase.

\section{Conclusions}

In summary, our field is at an interesting juncture where we can acknowledge and integrate two highly relevant and important research strands addressing the underlying and complex biological sleep and circadian processes alongside, rather than in isolation from, the social and interactive processes where communication is an important driver and motivator for use, which can have particular impact at night. We know from our work that FOMO [30] and exclusion, vigilance and obligation [33••] are all drivers for our adolescents to engage in night-time social media use and therefore we must acknowledge these cognitive factors alongside the physiological processes to understand the effect of light within the context of real world device use. Moving forward in the current research climate, we can meet these obligations through collaborations across research specialities and share data, skills and knowledge to build a comprehensive model of adolescent sleep and social interaction.

\section{Compliance with Ethical Standards}

Human and Animal Rights Informed Consent This article does not contain any studies with human or animal subjects performed by any of the authors.

Conflict of Interest Heather Cleland Woods and Holly Scott each declare no conflict of interest.
Open Access This article is distributed under the terms of the Creative Commons Attribution 4.0 International License (http:// creativecommons.org/licenses/by/4.0/), which permits unrestricted use, distribution, and reproduction in any medium, provided you give appropriate credit to the original author(s) and the source, provide a link to the Creative Commons license, and indicate if changes were made.

\section{References}

Papers of particular interest, published recently, have been highlighted as:

-• Of major importance

1. Jenni OG, Achermann P, Carskadon MA. Homeostatic sleep regulation in adolescents. Sleep. 2005;28(11):1446-54.

2. Anderson M, Jiang J. Teens, Social Media \& Technology 2018. 2018. https://www.pewinternet.org/2018/05/31/teens-socialmedia-technology-2018/.

3. Crowley SJ, Wolfson AR, Tarokh L, Carskadon MA. An update on adolescent sleep: new evidence informing the perfect storm model. J Adolesc. 2018;67:55-65. https://doi.org/10.1016/j.adolescence. 2018.06.001.

4. Carskadon MA. Sleep in adolescents: the perfect storm. Pediatr Clin N Am. 2011;58(3):637-47. https://doi.org/10.1016/j.pcl. 2011.03.003.

5. Wittmann M, Dinich J, Merrow M, Roenneberg T. Social jetlag: misalignment of biological and social time. Chronobiol Int. 2006;23(1-2):497-509. https://doi.org/10.1080/ 07420520500545979.

$6 . \bullet$ UK House of Committee Select Committee. Impact of social media and screen-use on young people's health. 2018. This UK government report does not react to popular media portrayal of the screentime debate but outlines a sensible overview of the evidence presented with acheiveable recommendations.

7. Souman JL, Tinga AM, te Pas SF, van Ee R, Vlaskamp BNS. Acute alerting effects of light: a systematic literature review. Behav Brain Res. 2018;337:228-39. https://doi.org/10.1016/j.bbr.2017.09.016.

8. Reinberg AE, Touitou Y. Synchronization and dyschronism of human circadian rhythms. Pathologie-biologie. 1996;44(6):487-95.

9. Touitou Y. Internal clock desynchronization, light and melatonin. Bull Acad Natl Med. 2011;195(7):1527-46 discussion 47-9.

10. Cajochen C, Frey S, Anders D, Spati J, Bues M, Pross A, et al. Evening exposure to a light-emitting diodes (LED)-backlit computer screen affects circadian physiology and cognitive performance. J Appl Physiol. 2011;110(5):1432-8. https://doi.org/10.1152/ japplphysiol.00165.2011.

11. Executive HaS. (2011) Changes in shift work patterns over the last ten 464 years (1999 to 2009). http://www.hse.gov.uk/research/ rrpdf/rr887.pdf

12. Technology TPOoSa. (2018) Shift work, sleep and health: Houses of Parliament. https://researchbriefings.parliament.uk/ ResearchBriefing/Summary/POST-PN-0586

13. Chang A-M, Aeschbach D, Duffy JF, Czeisler CA. Evening use of light-emitting eReaders negatively affects sleep, circadian timing, and next-morning alertness. Proc Natl Acad Sci. 2015;112(4): 1232-7. https://doi.org/10.1073/pnas.1418490112.

14. Rideout, V., Foehr, U., Roberts, D. (2010) Generation M2: media in the lives 472 of 8 to 18 -year-olds.: Kaiser Family Foundation Study. https://www.kff.org/other/poll-finding/report-generation-m2media-in-the-lives/ 
15. Orben A, Przybylski AK. The association between adolescent wellbeing and digital technology use. Nat Hum Behav. 2019;3(2):17382. https://doi.org/10.1038/s41562-018-0506-1.

16. Touitou Y, Touitou D, Reinberg A. Disruption of adolescents' circadian clock: the vicious circle of media use, exposure to light at night, sleep loss and risk behaviors. J Physiol Paris. 2016;110(4 Pt B):467-79. https://doi.org/10.1016/j.jphysparis.2017.05.001.

17. Greenwood S, Perrin A, Duggan M. Social Media Update. (2016) Pew Research Center. http://assets.pewresearch.org/wp-content/ uploads/sites/14/2016/11/10132827/PI 2016.11.11 Social-MediaUpdate FINAL.pdf

18. Scott, H., Biello, S.M., Woods, H.C. (2019) Social media use and adolescent sleep outcomes: cross-sectional findings from the millennium cohort study. https://doi.org/10.31234/osf.io/z7kpf

19. Avenevoli S, Swendsen J, He JP, Burstein M, Merikangas KR. Major depression in the national comorbidity survey-adolescent supplement: prevalence, correlates, and treatment. J Am Acad Child Adolesc Psychiatry. 2015;54(1):37-44 e2. https://doi.org/ 10.1016/j.jaac.2014.10.010.

20. Kelly Y, Zilanawala A, Booker C, Sacker A. Social media use and adolescent mental health: findings from the UK millennium cohort study. EClinicalMedicine. 2018;6:59-68. https://doi.org/10.1016/j. eclinm.2018.12.005.

21. Carr CT, Hayes RA. Social media: defining, developing, and divining. Atl J Commun. 2015;23(1):46-65. https://doi.org/10.1080/ 15456870.2015.972282.

22.• Bartel K, Gradisar M. New directions in the link between technology use and sleep in young people. In: Nevšímalová S, Bruni O, editors. Sleep disorders in children. Cham: Springer International Publishing; 2017. p. 69-80. This review introduces a new perspective on the screens at night debate achnowledging the need to appreciate all influencing factors that can impact sleep especially in our adolescents.

23. Chein J, Albert D, O'Brien L, Uckert K, Steinberg L. Peers increase adolescent risk taking by enhancing activity in the brain's reward circuitry. Dev Sci. 2011;14(2):F1-10. https://doi.org/10.1111/j. 1467-7687.2010.01035.x.

24. Somerville LH, Hare T, Casey BJ. Frontostriatal maturation predicts cognitive control failure to appetitive cues in adolescents. $\mathrm{J}$
Cogn Neurosci. 2011;23(9):2123-34. https://doi.org/10.1162/jocn. 2010.21572 .

25. Bowler J, Bourke P. Facebook use and sleep quality: light interacts with socially induced alertness. Br J Psychol. 2018. https://doi.org/ 10.1111/bjop.12351.

26. Horne JA, Donlon J, Arendt J. Green light attenuates melatonin output and sleepiness during sleep deprivation. Sleep. 1991;14(3): 233-40.

27. McNee, S and Woods, H.C. (2019) Pre-sleep cognitive influence of night-time social media use and social comparison behaviour in young women. https://doi.org/10.31234/osf.io/n9txa

28. Festinger L. A theory of social comparison processes. Hum Relat. 1954;7(2):117-40.

29. Nicassio PM, Mendlowitz DR, Fussell JJ, Petras L. The phenomenology of the pre-sleep state - the development of the pre-sleep arousal scale. Behav Res Ther. 1985;23(3):263-71. https://doi. org/10.1016/0005-7967(85)90004-X.

30. Scott H, Woods HC. Fear of missing out and sleep: cognitive behavioural factors in adolescents' nighttime social media use. J Adolesc. 2018;68:61-5. https://doi.org/10.1016/j.adolescence. 2018.07.009.

31. Owens J. Insufficient sleep in adolescents and young adults: an update on causes and consequences. Pediatrics. 2014;134(3): E921-E32. https://doi.org/10.1542/peds.2014-1696.

32. Tavernier R, Willoughby T. Sleep problems: predictor or outcome of media use among emerging adults at university? J Sleep Res. 2014;23(4):389-96. https://doi.org/10.1111/jsr.12132.

33. Scott, H., Biello, S. M., Woods, H.C. (2018) Identifying drivers for bedtime social media use despite sleep costs: the adolescent perspective. https://doi.org/10.31234/osf.io/2xb36

Publisher's Note Springer Nature remains neutral with regard to jurisdictional claims in published maps and institutional affiliations. 